\section{Endothelial injury, F-actin and vitamin D binding protein after hematopoietic stem cell transplant and association with clinical outcomes}

\author{
Nathan Luebbering, ${ }^{1,2}$ Sheyar Abdullah, ${ }^{1,2}$ Dana Lounder, ${ }^{1,2}$ Adam Lane, ${ }^{1,2}$ \\ Nikhil Dole, ${ }^{1,2}$ Jeremy Rubinstein,, Martin Hewison, ${ }^{1,2}$ Nicholas Gloude, ${ }^{1,2}$ \\ Sonata Jodele, ${ }^{1,2}$ Kitty MR Perentesis, ${ }^{1,2}$ Kelly Lake, ${ }^{1,2}$ Bridget Litts, ${ }^{1,2}$ \\ Alexandra Duell, ${ }^{1,2}$ Christopher E. Dandoy ${ }^{1,2}$ and Stella M. Davies ${ }^{1,2}$
}

${ }^{1}$ Division of Bone Marrow Transplantation and Immune Deficiency, Cincinnati Children's Hospital Medical Center Cincinatti, OH, USA; ${ }^{2}$ Department of Pediatrics, University of Cincinnati, Cincinnati, $\mathrm{OH}, \mathrm{USA}$ and ${ }^{3} \mathrm{~S} c h o o l$ of Clinical and Experimental Medicine, College of Medical and Dental Sciences, University of Birmingham, Birmingham, UK

\section{ABSTRACT}

E ndothelial injury after hematopoietic stem cell transplant is an important initiating factor for early transplant toxicities of thrombotic microangiopathy and acute graft versus host disease. We hypothesized that release of the angiopathic molecule filamentous actin (F-actin) from hematopoietic cells lysed during conditioning prior to stem cell transplant would be associated with clinical outcomes. We detected F-actin in the blood of $52 \%$ of stem cell transplant recipients in the first 14 days after transplant, and children with detectable F-actin had a significantly elevated risk of thrombotic microangiopathy $(P=0.03)$ and non-relapse mortality $(P=0.04)$. F-actin is cleared from the circulation by vitamin $\mathrm{D}$ binding protein (VDBP) so we expected that higher levels of VDBP would improve outcomes. In a cohort of 190 children receiving an allogeneic transplant, risk of thrombotic microangiopathy was reduced in those with serum concentrations of VDBP above the median at day $30(10 \%$ vs. $31 \%, P=0.01)$, and graft versus host disease and non-relapse mortality were reduced in those with levels above the median at day 100 (3\% vs. $18 \%, P=0.04$ and $0 \%$ vs. $15 \%, P=0.002$ ). Western blot analyses demonstrated actin-VDBP complexes in the blood, which cleared by day 21-28. Our data support modulation of cytokine secretion and macrophage phenotype by VDBP later after transplant. Taken together, our data identify an association between Factin, a mediator of endothelial damage, and VDBP, an actin scavenger, as modifiers of risk of clinical consequences of endothelial injury.

\section{Introduction}

Chemotherapy and radiation are used in very high doses to purposely eradicate an entire organ, the hematopoietic system, in situ, inside a human body prior to hematopoietic stem cell transplant (HSCT). This is a bold endeavour which results in significant toxicity, most notably in the first 4 weeks after transplant, when morbidity and mortality are high. Biomarker data support the hypothesis that early events, in the first 14 days after transplant, set the scene for later adverse events such as transplant-associated thrombotic microangiopathy (TA-TMA) and graft versus host disease (GvHD) which occur 21-60 days after HSCT. ${ }^{1}$ Endothelial injury seems to be a key event in the initiation of TA-TMA and GvHD, and contributes significantly to organ injury and death. ${ }^{2-11}$ We set out to identify possible mechanisms of endothelial injury, and to identify potential factors that ameliorate injury and modify the individual risk of TA-TMA and GvHD.

Cell lysis leads to the release of toxic intracellular molecules into the circulation, including filamentous actin (F-actin) and nucleotides such as ATP, that are usually
Haematologica 2021

Volume 106(5):1321-1329

\section{Correspondence:}

STELLA M. DAVIES

stella.davies@cchmc.org

Received: July 25, 2019.

Accepted: March 19, 2020.

Pre-published: April 2, 2020

https://doi.org/10.3324/haematol.2019.233478

(C)2021 Ferrata Storti Foundation

Material published in Haematologica is covered by copyright. All rights are reserved to the Ferrata Storti Foundation. Use of published material is allowed under the following terms and conditions:

https://creativecommons.org/licenses/by-nc/4.0/legalcode. Copies of published material are allowed for personal or internal use. Sharing published material for non-commercial purposes is subject to the following conditions: https://creativecommons.org/licenses/by-nc/4.0/legalcode, sect. 3. Reproducing and sharing published material for commercial purposes is not allowed without permission in writing from the publisher. 
present in low concentrations outside the cell. ${ }^{12}$ Actin is the most abundant protein in the human body and exists in two forms- monomeric or globular actin (G-actin) and a polymeric form, F-actin. F-actin is known to be angiopathic, and an effective actin scavenger system exists to rapidly clear F-actin that enters the circulation. ${ }^{13}$ Extracellular Factin is depolymerized into G-actin by plasma gelsolin. Monomeric G-actin then binds to the highly abundant circulating protein, vitamin $\mathrm{D}$ binding protein (VDBP), and the actin-VDBP complex is removed from the circulation by the reticulo-endothelial system, primarily in the liver. ${ }^{14}$ VDBP has been shown to be consumed in other clinical circumstances of significant cell lysis such as acute liver failure and major trauma. ${ }^{15,16}$

VDBP is an abundant protein, related to albumin and $\alpha$-fetoprotein. VDBP is conserved throughout evolution, and no humans deficient in VDBP have been identified, indicating an important biological function for VDBP. VDBP is a highly polymorphic molecule, with more than 70 different alleles described, and the frequency of three major alleles, Gc1s, Gc1f and Gc2 varies by race. ${ }^{17} \mathrm{VDBP}$ transports the major circulating form of vitamin $D$, 25 -hydroxyvitamin $\mathrm{D}$, and other vitamin $\mathrm{D}$ metabolites in the circulation, but only about $4 \%$ of VDBP is conjugated to vitamin $\mathrm{D}$ metabolites, indicating other important functions for the molecule. A deglycosylated form of VDBP has been shown to have immunomodulatory activity, serving as a so-called "macrophage-activating factor", 18,19 but also demonstrating capacity for regulation of angiogenesis. ${ }^{20}$ These properties of VDBP, in addition to actin scavenging, have the potential to significantly modify outcomes of HSCT.

We hypothesized that the release of F-actin would be associated with adverse clinical outcomes of transplant,

Table 1. Demographics of patients included in assessment of clinical outcomes and vitamin $D$ binding protein, divided according to whether cases developed or did not develop ransplant-associated thrombotic microangiopathy.

\begin{tabular}{lccc}
\hline Variable & TA-IMA & No TA-TMA & $P$ \\
Sex & & & 0.88 \\
Male & 39 & 70 & \\
Female & 30 & 51 & \\
Age & $10.2(5.6-15.4)$ & $7.4(3.5-13.3)$ & 0.042 \\
\hline Race & & & \\
Caucasian & 58 & 105 & 0.67 \\
$\quad$ Non-Caucasian & 11 & 16 & \\
Preparative regimen intensity & & & 0.53 \\
Myeloablative & 43 & 81 & \\
Reduced intensity & 26 & 40 & \\
\hline Donor type & & & 0.75 \\
Related & 20 & 39 & \\
$\quad$ Unrelated & 49 & 82 & \\
Stem cell source & & & 0.32 \\
Bone marrow & 50 & 77 & \\
Peripheral blood stem cells & 17 & 35 & \\
Cord blood & 2 & 9 & \\
\hline HLA match & & & 0.25 \\
Match & & \\
Mismatch & 45 & 89 & \\
\hline
\end{tabular}

Patients demographics included in the assessment of clinical outcomes and vitamin D binding protein (VDBP), divided according to whether cases developed transplantassociated thrombotic microangiopathy (TA-TMA) or did not develop TA-TMA (No TA-TMA). HLA: human leukocyte antigen. and that higher levels of VDBP would be associated with improved outcomes after transplant. Our data indicate a significant and beneficial role for VDBP in HSCT which appears to involve both actin scavenging and modulation of the macrophage phenotype.

\section{Methods}

\section{Patients}

Patient samples were obtained from the Cincinnati Children's Hospital Medical Center HSCT repository, approved by the Institutional Review Board. Demographics of 190 patients included in the study are shown in Table 1. Careful attention was paid to prospective phenotyping for TA-TMA including monitoring for schistocytes, lactate dehydrogenase, haptoglobin, proteinuria, elevation of creatinine, new and excessive thrombocytopenia and anemia, as previously reported. ${ }^{21}$

\section{Ultra-high performance liquid chromatography}

Serum vitamin $\mathrm{D}$ metabolites by ultra-high performance liquid chromatography were determined as previously described. ${ }^{22}$

\section{Enzyme-linked immunosorbent assay}

Enzyme-linked immunosorbent assay (ELISA) testing serum VDBP levels were measured using a polyclonal antibody (Genway Bio, DM3741), and commercial ELISA kits were used to measure serum F-actin (MyBioSource, 702018), IL6 (R\&D Systems Quantikine, D6050), TNF (R\&D Systems Quantikine, DTA00C), IL10 (R\&D Systems Quantikine, D1000B), and gelsolin (LSBio, F22526).

\section{Calculation of bioavailable 25-hydroxyvitamin D}

Calculation of bioavailable 25-hydroxyvitamin D (25-OH-D) is described in the Online Supplementary Appendix in Dahl et al. ${ }^{17}$

\section{Vitamin D-binding protein genotyping genotyping}

Participants were genotyped for two common single-nucleotide polymorphisms (SNP) in the coding region of the VDBP gene (Applied Biosystems, rs4588 and rs7041). TaqMan SNP Genotyping assay protocol was performed following the manufacture's guidelines (Applied Biosystems, 4371355).

\section{Western blot analysis}

Serum proteins were electrophoresed under native conditions through a precast $10 \%$ polyacrylamide gel (Bio-Rad, \#456-1034) and transferred using conventional techniques. The polyvinylidene fluoride membrane was probed with a mouse monoclonal antibody against native full-length, human plasma derived, VDBP (1:4000, Abcam, ab23480), then incubated with an horse radish peroxidase conjugated, anti-mouse immunoglobulin G (IgG) antibody (1:5,000, Cell Signalling, 7076P2). Results were visualized by chemiluminescence (Amersham, RPN2235) using a BioRad ChemiDoc Touch imager. A positive actin-VDBP complex band $(\sim 250 \mathrm{kDa})$ was scored visually.

\section{Measurements of ATP levels}

Measurement of ATP levels by quantitative mass spectrometry is detailed in the Online Supplementary Appendix.

\section{RNA sequencing studies}

RNA sequencing (RNAseq) studies were performed as described previously. ${ }^{23}$ Briefly, normal peripheral blood mononuclear (PBMC) were incubated overnight with sera from two HSCT recipients, collected at days 0 and 100 (four wells total). RNA was 
extracted and submitted for RNAseq analysis. Gene expression at day 100 was compared with expression at day 0 .

\section{Glucose stress test}

Macrophage polarization was examined by measurement of extracellular acidification rate (ECAR) using Seahorse technology. Briefly, normal PBMC were incubated overnight with sera collect- ed at days 0 and 100 from two HSCT recipients. Cells were harvested and replated before performance of a glucose stress test and metabolic analysis.

\section{Vitamin D binding protein glycosylation studies}

Vitamin D binding protein glycosylation studies are detailed in the Online Supplementary Appendix.
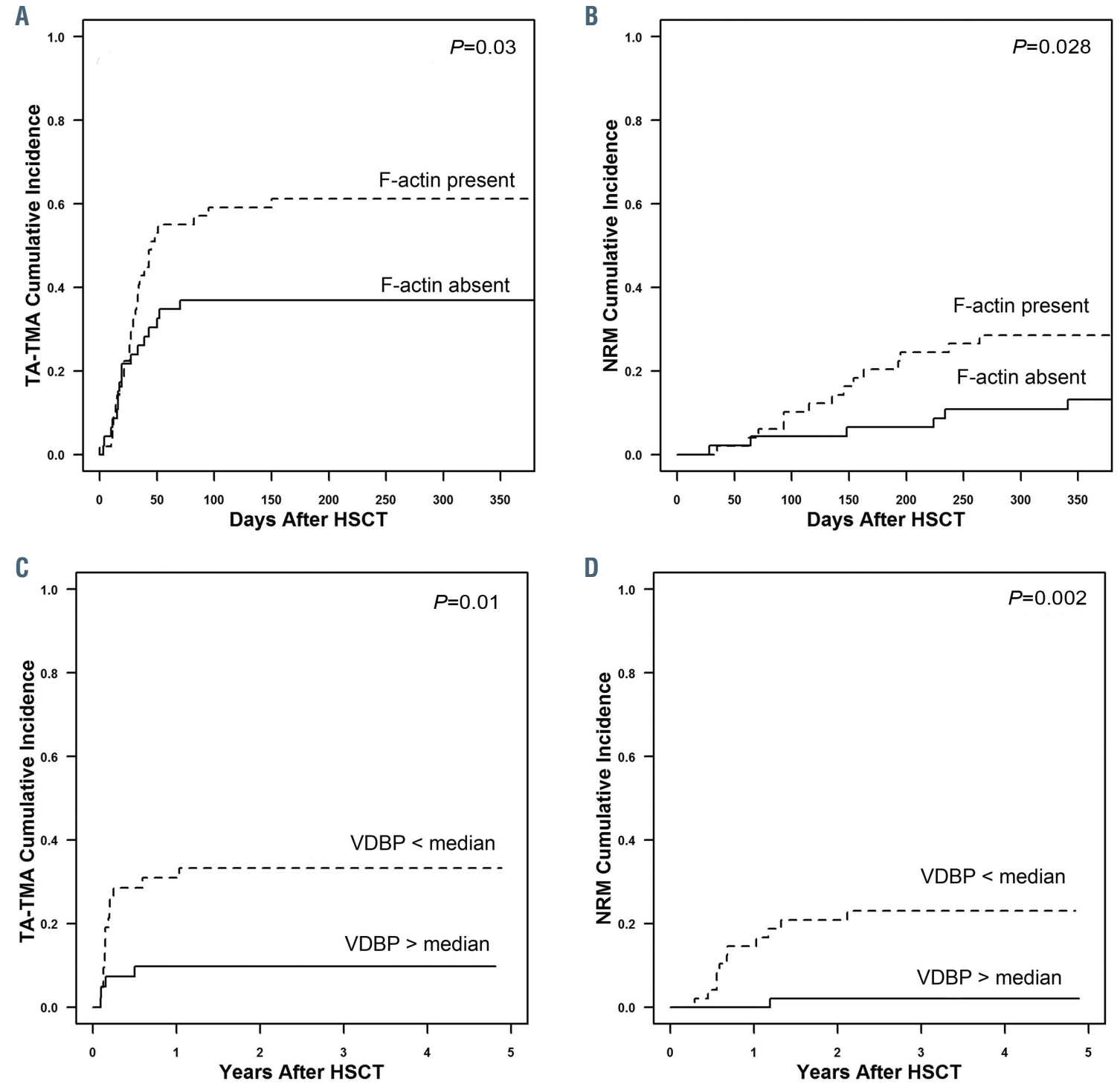

Figure 1. The association of detectable filamentous actin and vitamin D binding protein with clinical outcomes of hematopoietic stem cell transplant. (A) Cumulative incidence of transplant-associated thrombotic microangiopathy (TA-TMA) in hematopoietic stem cell transplant (HSCT) recipients with and without detectable filamentous actin (F-actin) $(61 \%$ vs. $37 \%, P=0.03)$. (B) Cumulative incidence of non-relapse mortality (NRM) in HSCT recipients with and without detectable F-actin (29\% vs. 13\%, $P=0.028)$. (C) Cumulative incidence of TA-TMA in HSCT recipients with vitamin D binding protein (VDBP) levels above and below the median $(10 \%$ vs. $31 \%, P=0.01)$. (D) Cumulative incidence of NRM in HSCT recipients with VDBP levels above and below the median $(0 \%$ vs. $15 \%, P=0.002)$.

Table 2. Outcomes of bone marrow transplant in pediatric hematopoietic stem cell transplant recipients according to vitamin D binding protein level at days 30 and 100 .

\begin{tabular}{|c|c|c|c|c|c|c|}
\hline Outcome & VDBP> Median & $\begin{array}{l}\text { Day } 30 \text { VDBP } \\
\text { VDBP<Median }\end{array}$ & P & VDBP> Median & $\begin{array}{l}\text { Day } 100 \text { VDBP } \\
\text { VDBP }<\text { Median }\end{array}$ & $P$ \\
\hline TA-TMA & $10 \%$ & $31 \%$ & 0.01 & $0 \%$ & $7 \%$ & 0.45 \\
\hline GvHD & $8 \%$ & $21 \%$ & 0.09 & $3 \%$ & $18 \%$ & 0.04 \\
\hline NRM & $8 \%$ & $10 \%$ & 0.1 & $0 \%$ & $15 \%$ & 0.002 \\
\hline
\end{tabular}

VDBP: vitamin D binding protein;TA-TMA: transplant-associated thrombotic microangiopathy; GvHD: graft versus host disease; NRM: non-relapse mortality 
A

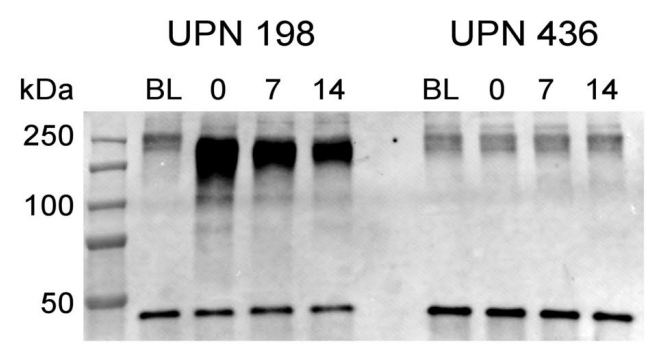

B

UPN 324

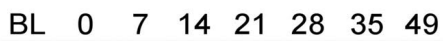

C

UPN 336
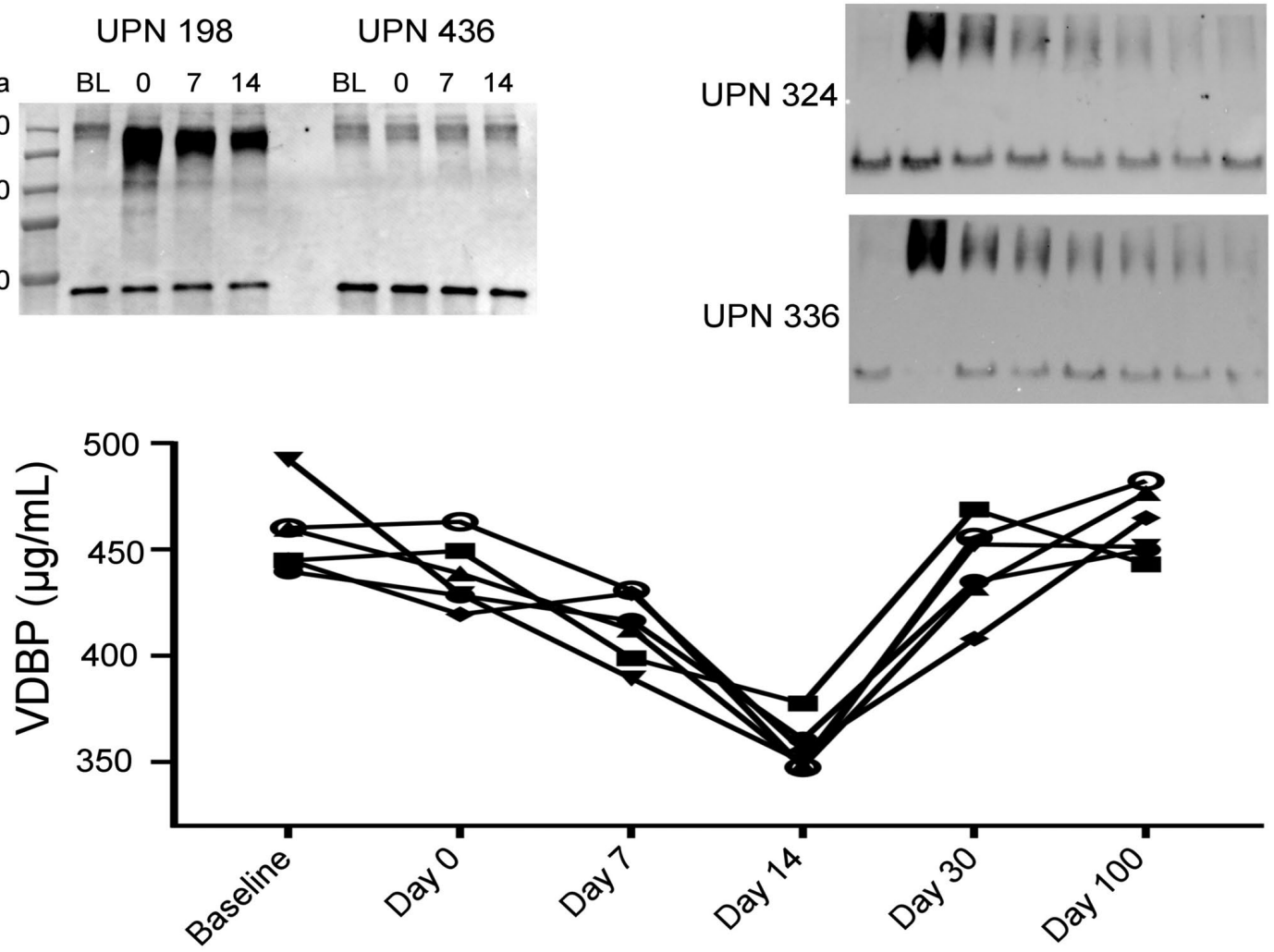

Figure 2. The formation and clearance of actin-vitamin D binding protein (VDBP) complexes after hematopoietic stem cell transplant (HSCT) and the impact of VDBP genotype on modulation of VDBP levels after HSCT. (A) Western blot probed for VDBP showing serum collected pre-transplant, and at days 0,7 , and 14 after transplant from two HSCT recipients. A lower molecular weight band corresponding to VDBP (unbound to filamentous actin) is seen in all samples including pre-transplant serum (baseline [BL]). However, in post-transplantation serum a larger slower migrating band was also observed corresponding to VDBP bound to actin collected on days 0, 7 and 14, demonstrating the presence of circulating actin-VDBP complex in samples from one patient, unique patient number (UPN) 198. Similar increase in levels of circulating actin-VDBP complex is not seen in patient UPN 436, illustrating inter-individual variability. BL: sample collected before the start of transplant. (B) Western blot probed for VDBP showing clearance of increased actin-VDBP complexes from the circulation, with complexes largely cleared by days 21-28 after HSCT in two different transplant recipients (UPN 324 and UPN 336). (C) VDBP levels according to genotype, measured at BL, days 0, 7, 14, 30 and 100 after HSCT, showing decreased levels at day 14 in all genotypes.

\section{Statistical analysis}

Categorical and continuous demographics are described by frequency (percent) and median (range), respectively. Time to event data, non-relapse mortality (NRM), GvHD and TA-TMA are described at 1 year and with cumulative incidence curves using the Kaplan-Meier method incorporating death as a competing risk. In both the day 30 and 100 analysis patients with an event preceding the date of measurement were excluded. This was done to ensure that the measured VDBP preceded the respective NRM, GvHD or TA-TMA event. Gray's method for competing risks was used to test for differences in time to event between groups. Glycan data are summarized by mean and levels were compared between day 0 and day 100 using a paired $t$-test.

\section{Results}

\section{Circulating filamentous actin is associated with clinical outcomes of hematopoietic stem cell transplant}

Our overall goal was to understand the mechanism of endothelial injury after HSCT. We hypothesized that F-actin is released into the circulation during lysis of hematopoietic cells caused by chemotherapy and radia- tion given prior to HSCT. F-actin is known to be toxic to endothelial cells and is generally not present in the circulation. First we looked for detectable F-actin in the circulation at three timepoints after HSCT (baseline, days 0, 7, 14) in an unselected cohort of 96 consecutive transplant recipients who consented to participate in our institutional HSCT sample repository and scored any finding of Factin as positive. Cases with no F-actin measured at any of the three timepoints were scored as negative. Fifty of 96 $(52 \%)$ cases analyzed had detectable F-actin. We then examined the impact of F-actin presence in the first 14 days of transplant on outcomes of transplant. We found higher risk of TA-TMA in those with F-actin in the circulation $(61 \%$ vs. $37 \%$ at 1 year, $P=0.03$, Figure $1 \mathrm{~A})$. Adjustment of the data for age and HLA-match yielded a $P$-value of 0.06 . Moreover, NRM was increased in those with F-actin in the circulation $(29 \%$ vs. $13 \%, P=0.028$, Figure 1B). Adjustment of the NRM data for age and human leukocyte antigen (HLA)-match yielded a $P$-value of 0.07 . In an analysis of factors that might modify likelihood of increased F-actin we found that $41 \%$ of recipients of myeloablative preparative regimen had detectable F-actin compared with $64 \%$ of recipients of reduced intensity conditioning $(P=0.04)$. 


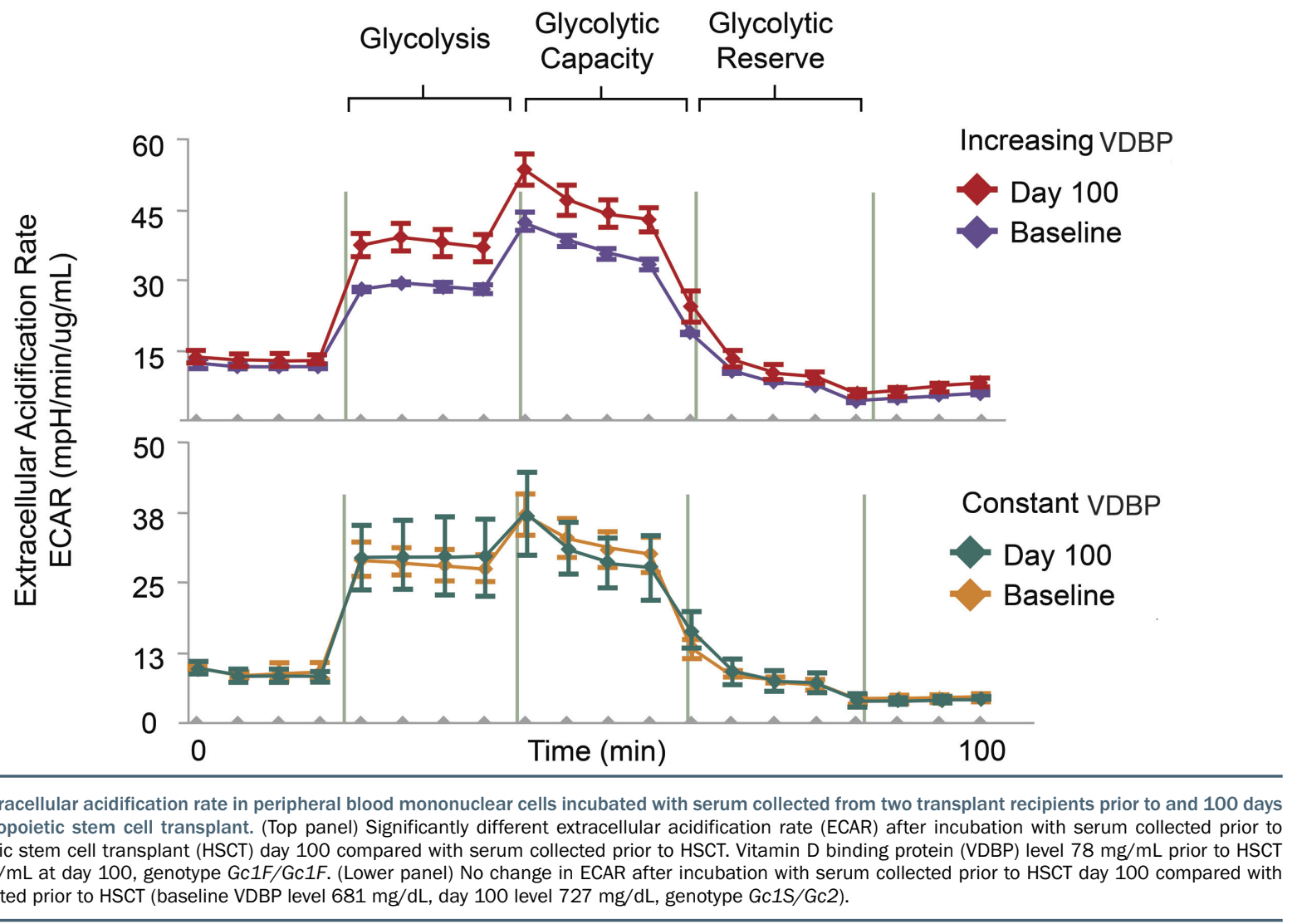

Circulating concentrations of vitamin D binding protein (VDBP) and VDBP genotype are associated with clinical outcomes of hematopoietic stem cell transplant

VBDP is known to function as an actin scavenger, removing angiopathic F-actin from the circulation. We measured VBDP levels at six timepoints after HSCT (baseline, days $0,7,14,30$ and 100) in an expanded cohort of 190 consecutive HSCT recipients. Demographics of the cohort are shown in Table 1.

VDBP levels prior to start of transplant, and at day 0 and day 7 had no impact on transplant outcomes. In contrast, a higher day 30 VDBP level was associated with lower occurrence of TA-TMA in the first year after HSCT (31\% vs. $10 \%, P=0.01$, Figure $1 \mathrm{C}$, Table 2). Adjustment of the data for TA-TMA risk factors of race and diagnosis yielded a $P$-value of 0.005 . The rate of GvHD at 1 year was also higher in those with a lower VDBP level, although this did not reach statistical significance $(21 \%$ vs. $8 \%, P=0.09$, Table 2). We then analyzed VDBP level at day 100, looking for associations with clinical events occurring between day 100 and 1 year (summarized in Table 2). NRM at 1 year was significantly higher in HSCT recipients with a VDBP level lower than the median at day 100 (15\% vs. $0 \%, P=0.002$, Table 2, Figure 1D). Adjustment of the data for NRM risk factors of age and HLA-match yielded a $P$-value of 0.015 . Moreover, GvHD at 1 year was more frequent in HSCT recipients with a VDBP level lower than the median at day 100 (18\% vs. $3 \%, P=0.04$, Table 2$)$. In contrast, rates of TA-TMA did not correlate with VDBP level at day 100 .

VDBP is a highly polymorphic protein, with three major alleles $(G c 1 F, G c 1 S$ and $G c 2)$ and a large number of rare
Table 3. Outcomes of hematopoietic stem cell transplant.

\begin{tabular}{lccccc} 
& $\begin{array}{c}\text { Number } \\
\text { of cases }\end{array}$ & TA-TMA & $P$ & NRM & $P$ \\
\hline Gc1S/Gc1S & 57 & $33 \%$ & & $12 \%$ & \\
Gc1F/Gc1F & 16 & $63 \%$ & & $13 \%$ & \\
\hline Gc2/Gc2 & 17 & $6 \%$ & & $0 \%$ & \\
& & & 0.001 & & 0.22 \\
\hline
\end{tabular}

Outcomes of hematopoietic stem cell transplant according to host vitamin D binding protein, only in cases with a homozygous genotype. TA-TMA: transplant-associated thrombotic microangiopathy; NRM: non-relapse mortality.

minor alleles. We asked whether the VDBP genotype influenced transplant outcomes by comparing transplant outcomes in HSCT recipients with the three homozygous genotypes Gc1F/Gc1F, Gc1S/Gc1S and Gc2/Gc2 (Table 3). Genotyping was performed using host DNA stored prior to HSCT as VDBP is primarily synthesized in the liver. We analyzed homozygous cases only to maximize our chance of seeing biological differences related to the genotype (90 total cases, $57 \mathrm{Gc1s} / \mathrm{Gc1S}, 16 \mathrm{Gc1F} / \mathrm{Gc1F}$ and $17 \mathrm{Gc2} / \mathrm{Gc2}$ ). The data show a significant difference in frequency of TATMA according to the genotype with the highest frequency seen in $G c 1 F / G c 1 F$ individuals and the lowest in those with a Gc2/Gc2 genotype. NRM frequencies were not statistically different.

\section{Actin-vitamin D binding protein complex formation in hematopoietic stem cell transplant patients}

We next investigated the kinetics of actin-VDBP complexes in the circulation. Typically, F-actin is cleared rapidly from the circulation by depolymerization by gelsolin, 


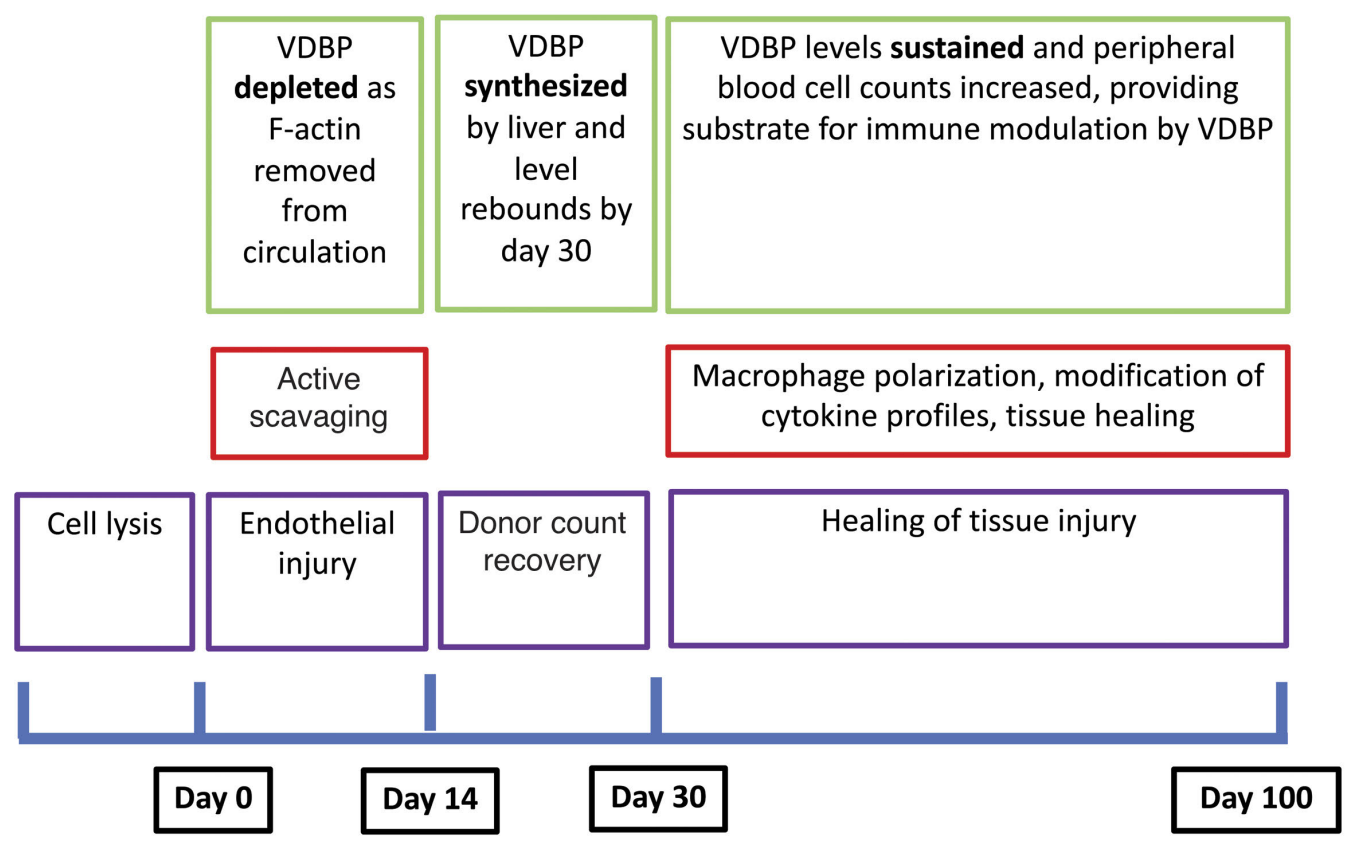

Figure 4.Vitamin D binding protein function after hematopoietic stem cell transplant. Schematic of proposed vitamin D binding protein (VDBP) activity during hematopoietic stem cell transplant, with predominant effect early after transplant being actin scavenging with later effects on macrophage polarization and tissue healing.

followed by binding of monomeric actin to VDBP. The actin-VDBP complex is then removed from the circulation by the reticulo-endothelial system with a reported half-life of 30 minutes. We first examined actin-VDBP complex formation by western blot analysis of serum VDBP after running a native gel to identify mobility changes of serum VDBP due to binding of actin released by cell lysis. Results for analysis of serum VDBP from one patient (unique patient number [UPN] 198) showed the presence of a lower molecular weight band corresponding to VDBP (unbound to F-actin) in all samples including pre-transplant serum (baseline [BL]). However, in post-transplantation serum a larger slower migrating band was also observed corresponding to VDBP bound to actin (Figure $2 \mathrm{~A})$. There was variability in the presence of complex seen after transplant, with large amounts of complex seen in some cases, while less or none was seen in others. For example one patient, UPN 198, had a large amount of complex present post-transplant, while a second patient, UPN 436, had modest and similar amounts of complex present at all timepoints examined (Figure 2A).

We considered whether the presence of a large amount of complex on day 0 might be associated with inferior outcomes, as has been suggested by a prior animal study. ${ }^{24}$ Western blot analysis of VDBP was carried out using serum from 93 transplant recipients, and each blot was scored as positive (as seen in UPN 198, Figure 2A left panel) or negative (as seen in UPN 436, Figure 2A right panel) for the presence of elevated-actin-VDBP complex. We found no association between the presence of elevated actin- VDBP complex and TA-TMA, (35\% vs. $40 \%$, $P=0.67), \mathrm{GvHD}$ ( $35 \%$ vs. $26 \%, P=0.39)$ or NRM $(5 \%$ vs. $10 \%, P=0.66)$. We also considered whether the amount of complex simply reflected the level of VDBP but found no such association $(P=0.17)$.

VDBP bound to F-actin is removed from the circulation by the reticuloendothelial system, so that serum levels of actin-VDBP complex are likely to change post-transplant. We therefore examined the kinetics of clearance of complex in a patient with a large amount of complex further by examining later timepoints after infusion of stem cells
(Figure 2B). Data show that levels of actin-VDBP complex returned to baseline by 21-28 days after transplant. Further analysis showed a temporal decline in serum VDBP levels at day 14 post-transplantation, common to all patients and all VDBP genotypes, with a rebound back to previous levels by day 30 post HSCT (Figure 2C).

Gelsolin also participates in actin scavenging by VDBP, serving to depolymerize F-actin to monomeric G-actin. We measured plasma gelsolin levels on day 7 after transplant, and found that levels above the median were associated with increased risk of TA-TMA $(53 \%$ vs. $32 \%$, $P=0.03$ ), but gelsolin levels were not associated with any other outcome. We also hypothesized that endothelial toxicity may also be related to the release of ATP into the circulation together with F-actin, but found no evidence of an increase in circulating ATP after HSCT. Extracellular ATP increased in only 1 of 4 HSCT recipients, in contrast to our hypothesis that extracellular ATP would increase markedly in most cases at the time of absolute neutrophil count nadir (time of maximum cell lysis) after HSCT (Online Supplementary Figure S1). The main cause of death in those with no detectable F-actin was relapse, in contrast to those with detectable F-actin in whom the main causes of death was GvHD (in which endothelial injury is implicated) (Online Supplementary Table S2).

\section{Vitamin B binding protein functions as a macrophage-activation factor}

Our findings regarding clearance of F-actin by VDBP provide a plausible mechanism for a role for VDBP in protection against endothelial injury and early toxicities of transplant such as TA-TMA and GvHD. However, as VDBP can also function as a macrophage activating factor, we investigated whether higher levels of VDBP functioned to modify cytokine production by immune cells later after transplant. ${ }^{18,19}$ PBMC from a normal healthy volunteer were incubated overnight with serum from 18 different HSCT recipients at four different timepoints. Supernatants from these cells were then assayed for key cytokines and the concentrations of these cytokines were correlated with serum VDBP, total 25-hydroxyvitamin D 
Table 4. Mononuclear cell analysis. Mononuclear cells from the same normal donor were incubated overnight in individual wells with individual sera from 18 children receiving hematopoietic stem cell transplant (HSCT), collected at days 0, 30, 60 and 100 after HSCT.

\begin{tabular}{|c|c|c|c|c|c|c|c|}
\hline & & \multicolumn{2}{|c|}{ IL6 } & \multicolumn{2}{|c|}{ TNF $\alpha$} & \multicolumn{2}{|c|}{ IL10 } \\
\hline & & $r^{2}$ & $P$ & $r^{2}$ & $P$ & $r^{2}$ & $P$ \\
\hline \multirow[t]{3}{*}{ Day 0} & VDBP & 0.14 & 0.58 & 0.079 & 0.75 & 0.16 & 0.52 \\
\hline & 25-OH Vit D & 0.063 & 0.80 & 0.21 & 0.41 & 0.20 & 0.43 \\
\hline & Free Vit D & 0.077 & 0.76 & 0.046 & 0.85 & -0.061 & 0.81 \\
\hline \multirow[t]{3}{*}{ Day 30} & VDBP & -0.07 & 0.83 & -0.27 & 0.37 & 0.10 & 0.73 \\
\hline & $25-\mathrm{OH}$ Vit D & -0.32 & 0.28 & -0.11 & 0.71 & 0.36 & 0.23 \\
\hline & Free Vit D & -0.16 & 0.60 & 0.11 & 0.72 & 0.21 & 0.48 \\
\hline \multirow[t]{3}{*}{ Day 60} & VDBP & 0.53 & 0.028 & 0.52 & 0.027 & -0.65 & 0.0036 \\
\hline & $25-\mathrm{OH}$ Vit D & 0.19 & 0.45 & 0.23 & 0.35 & -0.30 & 0.22 \\
\hline & Free Vit D & 0.18 & 0.18 & 0.12 & 0.64 & -0.16 & 0.53 \\
\hline \multirow[t]{3}{*}{ Day 100} & VDBP & 0.7 & 0.0013 & 0.28 & 0.27 & -0.46 & 0.05 \\
\hline & 25-OH Vit D & 0.47 & 0.05 & -0.12 & 0.63 & -0.40 & 0.10 \\
\hline & Free Vit D & -0.28 & 0.26 & -0.63 & 0.005 & 0.24 & 0.33 \\
\hline
\end{tabular}

Cytokine levels were measured by enzyme-linked immunosorbent assay in supernatant from each well. Cytokine levels were correlated with levels of vitamin $\mathrm{D}$ binding protein (VDBP), 25-hydroxy vitamin D (25-OH Vit D) and free vitamin D (Free Vit D); IL6; interleukin 6, IL10: interleukin 10; TNF $\alpha$ : tumor necrosis factor $\alpha$.

(25-OH-D) and free 25-OH-D levels (Table 4). Data show no significant correlation between VDBP, and total or free 25-OH-D and any cytokine at days 0 and 30. In contrast, the day 60 data show a significant positive association between VDBP and IL- 6 and TNF $\alpha$, and a significant negative association between VDBP and IL-10. The association between VDBP and IL- 6 and IL-10 was also seen with sera collected at day 100. A positive association between $25-\mathrm{OH}-\mathrm{D}$ and IL-6 and a negative association between free 25-OH-D and TNF $\alpha$ was also observed at day 100.

These data suggest that serum VDBP and its cargo, 25-OH-D, have the potential to modify immune cell function, but this effect was only evident at later time points following transplant. In an exploratory analysis, seeking direction as to the possible nature of this immune modulation we incubated PBMC from a normal volunteer overnight with serum from a person who had stable levels of VDBP at baseline and day 60 and with serum from a person in whom VDBP levels increased significantly at day 60 compared with baseline. Changes in the gene expression profile between the same cells conditioned with serum from these two distinct patients were compared using RNAseq, and the genes with the most notable change in expression are listed in the Online Supplementary Table S1. Observed changes in gene expression were consistent with altered macrophage function, consistent with the established potential for a deglycosylated form of VDBP to act as a macrophage activating factor. The RNAseq data, together with the change in the cytokine profile, supported a possible change in macrophage polarization as a consequence of exposure to VDBP after HSCT. We addressed this further by incubating CD14/16 expressing normal peripheral blood macrophages separately with serum collected from the same HSCT recipient at baseline (VDBP level 78) and at day 100 (VDBP level 907, genotype Gc1F/Gc1F) and from a second HSCT recipient in whom VDBP levels were more constant (baseline VDBP level 681, day 100 level 727, genotype Gc1S/Gc2). We then assessed macrophage polarization in the resulting cell cultures by measuring the ECAR during a glucose stress test performed using Seahorse technology (Figure 3). Data indicate altered macrophage polarization in cells incubated with serum containing a higher level of VDBP at day 100, but not in cells incubated with serum containing lower levels of VDBP at day 100.

VDBP macrophage activating activity has been reported to vary by genotype and by post-translational glycosylation. ${ }^{25-27}$ We hypothesized that changes in glycosylation of VDBP during the course of transplant might play a role in modifying macrophage polarization after HSCT. We tested this hypothesis by measuring glycosylation of VDBP in serum samples from two persons of differing VDBP genotype, collected at days 0 and 100 after HSCT (four samples total) to see if there were significant changes in glycosylation during recovery after HSCT (Online Supplementary Table S2). The only significant change in glycosylation between days 0 and 100 occurred at a single minor glycan $(\operatorname{HexNAc}(1) \operatorname{Hex}(1) \mathrm{NeuAc}(2))$, with no significant change seen in the major glycosylated glycan (HexNAc(1)Hex(1) $\operatorname{NeuAc}(1))$, suggesting that changes in glycosylation are likely not a major contributor to changes in immune modulation by VDBP.

\section{Discussion}

Endothelial injury immediately following HSCT is an important initiator of later complications such as TA-TMA and GvHD, which together are major causes of morbidity and mortality after HSCT. ${ }^{1}$ Effector mechanisms leading to damage to the endothelium have not been described, although direct injury by chemotherapy and injury secondary to viral reactivation are possible causes. In this study we show that release of the angiopathic molecule $\mathrm{F}$ actin into the circulation is associated with TA-TMA, an immediate clinical consequence of endothelial damage. Factin is typically not present in the circulation of healthy individuals who have low and relatively constant levels of cell turnover that do not exceed the capacity of the actin scavenger system for rapid removal of the harmful protein. ${ }^{12,13}$ Rapid lysis of the entire hematopoietic system during conditioning therapy for HSCT overwhelms actin scavenging, at least in some cases, and allows F-actin to remain in the circulation and cause damage. We also found that F-actin was more likely to be detected after a myeloablative than a reduced intensity preparative regi- 
men. We hypothesize that this finding may be due to the frequent use of myeloablative preparative regimens in our practice for children with leukemia who have received extensive prior chemotherapy while non-myeloablative regimens are used for children with non-malignant disorders who have not received chemotherapy. Children who have received prior chemotherapy are likely to have a reduced mass of hematopoietic cells to be lysed by the preparative regimen and hence have less release of F-actin into the circulation. Similar findings have been described in the settings of acute liver failure and massive trauma, and low VDBP levels predict poor outcome in both these clinical settings, in agreement with our findings in this study. ${ }^{15,16,28-30}$ Our findings differ from reports of acute liver failure in that HSCT recipients generally have healthy livers and we were able to see rapid rebound of VDBP levels after initial depletion.

In this paper we show for the first time that VDBP levels are associated with outcomes of HSCT. Our data show both an association between day 30 VDBP level and TATMA, and a strong association of day 100 level with NRM. Our data show a differential effect of the VDBP genotype on the early outcome of TA-TMA, with lower TA-TMA in Gc2 homozygous transplant recipients. A large number of prior epidemiological studies have identified associations between VDBP polymorphism and assorted chronic and frequent diseases, including chronic obstructive lung disease, ${ }^{31}$ diabetes mellitus ${ }^{32}$ and coronary artery disease, ${ }^{33,34}$ supporting some functional importance to the variants, although reproducibility of association studies is mixed. Despite the many association studies, there have been few functional studies that offer insight into potential mechanisms. Gc1 and Gc2 alleles differ by a single amino acid and by differential post-translational glycosylation. Gc1s and Gc1f alleles differ by a single amino acid but are similarly glycosylated. The functional consequences of these changes are poorly understood, although in general Gc2 homozygotes do have modestly reduced VDBP levels. We speculate that the difference in TA-TMA risk by genotype might relate to differential binding of actin by genotype, or differential clearance of actin-VDBP complexes. This question is a subject of ongoing investigation.

We recognize that the frequency of TA-TMA reported from our center is notably higher than in other reports. We reported a prospective study of systematic screening for TA-TMA and identified an incidence of TA-TMA of $39 \% .{ }^{21}$ It is important to stress that this incidence includes a majority of cases with biological evidence of TA-TMA that will never require clinical treatment due to absence of organ injury. Such cases would not be discerned in the absence of prospective detailed screening of markers such as lactate dehydrogenase and schistocytes, at our center or at others. In the same work we describe an incidence of TA-TMA with evidence of organ injury worthy of consideration of treatment of approximately $15 \%$. The generalizability of these data is an important question. Our center treats a disproportionate number of children with immune deficiency disorders such as hemophagocytic lymphohistiocytosis and Fanconi anemia that are associated with increased risk of TA-TMA, amplifying incidence at our site. We have addressed the question of incidence of TA-TMA at other centers directly with collaborators by performing a multi-center study of prospective screening for TA-TMA, using the same screening algorithms. This
PBMTC/PALISI sponsored study included 13 diverse (large and small, academic and community) pediatric transplant centers transplanting for largely malignant disease (personal communication, CE Dandoy, February 2020). Six hundred and fourteen children were studied and $18 \%$ met criteria for TA-TMA, demonstrating the importance of prospective observations to identify the frequency of TMA. We believe that these data support the high frequency of TA-TMA consistently observed at our center, although a significant proportion of cases observed in this way do not merit changes in therapy. Clinical context must be carefully considered in initiating and therapy for TA-TMA.

VDBP functions as an actin scavenger, and in this role has been shown to be key in recovery from massive cell lysis occurring in settings such as acute liver failure and massive trauma. Higher levels of VDBP are associated with improved survival in those clinical settings, likely because VDBP serves to remove the angiopathic molecule F-actin, similar to the findings in our study. F-actin is clearly identified as directly angiopathic in previous literature. Surprisingly, however, direct infusion of monomeric actin into $d b p$ null mice showed increased pulmonary toxicity in the $d b p$ deficient compared to $d b p$ sufficient animals. This study raises concern that the angiopathic molecule might be the actin-VDBP complex, and not unbound free actin. This distinction is important because if unbound free actin is the toxic molecule, one might speculate that infusion of additional VDBP around the time of transplant would be beneficial. VDBP is an abundant protein that could be prepared and infused, in a similar manner to albumin. Our human data show consistent advantage for a higher level of VDBP, in contrast to the mouse study, and no association of adverse outcomes with high levels of circulating actin-VDBP complex. These disparate findings may reflect important differences between the mouse model and clinical HSCT.

We found considerable variation in the amount of actinVDBP complex present at day 0 (the day of stem cell infusion, after completion of conditioning therapy), between individuals. The amount of complex present at day 0 could be influenced by many variables, including the amount of hematopoiesis present to be lysed (high in active leukemia, low in marrow failure), the VDBP level, the efficiency of the reticulo-endothelial system in removing complex and of the liver in replacing VDBP and the intensity and speed of delivery of the preparative regimen so variability is perhaps not surprising. It is important to recognize that studies in human disease are challenged by heterogeneity of cases, often small sample size, and need for assessment in multiple sample sets to validate findings and establish whether observations are generalizable to all populations.

The benefit of VDBP appears to last longer than the early weeks after transplant when endothelial injury occurs, and in this context appear independent of vitamin D transport. A higher day 100 VDBP level was associated with significantly improved NRM, a time long after lysis of the host hematopoietic system and clearance of F-actin, but a critical time for recovery of the donor immune system and for ongoing healing of epithelial injury. VDBP is known to act as a so-called "macrophage activating" factor, so we hypothesized that VDBP levels might favorably influence immune recovery and tissue healing in the later weeks after HSCT. We found changes in cytokine secre- 
tion and changes in expression of genes related to macrophage polarization associated with VDBP levels. For example, marked up-regulation of ADORA3 would promote IL6 production which would favor epithelial healing. We propose that higher levels of VDBP at later timepoints serve to modulate immune recovery, while at early timepoints, prior to donor cell recovery, there are few if any macrophages to modulate and so no effect is apparent.

Figure 4 shows in schematic form our proposal for VDBP function after HSCT. We envisage that in the early days after transplant VDBP functions as an actin scavenger, protecting the endothelium and preventing early complications such as TA-TMA. VDBP is depleted in this process, but rebounds around day 30. VDBP has little opportunity to act as an immunomodulator in the early weeks after HSCT as donor cell counts have not yet recovered. Between days 30 and 60 after HSCT donor cell counts recover and tissue healing takes place, and VDBP can modify macrophage phenotype to favor tissue healing. Taken together, these data indicate important benefit for higher levels of VDBP after HSCT, and further work will clarify whether there would be potential benefit from strategies to increase or infuse additional VDBP.

\section{Disclosures}

SMD has served as a consultant for Novartis and has received research support from Prolacta and Alexion pharmaceuticals; SJ has received research support from Alexion pharmaceuticals; NL and SMD are holders of a provisional US patent regarding vitamin D-binding protein as a modifier of transplant outcomes.

\section{Contributions}

$N L, S A, D L, N D, J R, N G, E M R P, K L, B L, A D$ performed experiments and reviewed and approved the manuscript; $A L$ performed statistical analyses; NL, MH, SJ, CED and SMD designed experiments, analyzed and interpreted data and reviewed and approved the manuscript.

\section{Acknowledgments}

We gratefully acknowledge financial support from the Mueller Family Foundation and technical assistance from Edith Janssen, PhD and Joseph Qualls, PhD.

\section{Funding}

This work was supported by R01 HD093773 to SJ and $S M D$

\section{References}

1. Rotz SJ, Dandoy CE, Davies SM. ST2 and endothelial injury as a link between GVHD and Microangiopathy. N Engl J Med. 2017; 376(12):1189-1190.

2. Jodele S, Dandoy CE, Myers K, et al. High-dose carboplatin/etoposide/melphalan increases risk of thrombotic microangiopathy and organ injury after autologous stem cell transplantation in patients with neuroblastoma. Bone Marrow Transplant. 2018; 53(10):1311-1318.

3. Gloude NJ, Khandelwal P, Luebbering N, et al. Circulating dsDNA, endothelial injury, and complement activation in thrombotic microangiopathy and GVHD. Blood. 2017; 130(10):1259-1266.

4. Jodele S, Laskin BL, Dandoy CE, et al. A new paradigm: Diagnosis and management of HSCT-associated thrombotic microangiopathy as multi-system endothelial injury. Blood Rev. 2015;29(3):191-204.

5. Tichelli A, Gratwohl A. Vascular endothelium as 'novel' target of graft-versus-host disease. Best Pract Res Clin Haematol. 2008; 21(2):139148.

6. Luft T, Benner A, Jodele S, et al. EASIX in patients with acute graft-versus-host disease: a retrospective cohort analysis. Lancet Haematol. 2017;4(9):e414-e423.

7. Luft T, Benner A, Terzer T, et al. EASIX and mortality after allogeneic stem cell transplantation. Bone Marrow Transplant. 2020; 55(3):553-561.

8. Rachakonda SP, Dai H, Penack O, et al. Single nucleotide polymorphisms in CD40L predict endothelial complications and mortality after allogeneic stem-cell transplantation. J Clin Oncol. 2018; 36(8):789-800.

9. Radujkovic A, Dai H, Kordelas L, et al. Asymmetric dimethylarginine serum levels are associated with early mortality after allogeneic stem cell transplantation. Haematologica. 2019;104(4):827-834.

10. Carreras E, Diaz-Ricart M. The role of the endothelium in the short-term complications of hematopoietic SCT. Bone Marrow Transplant. 2011:46(12):1495-502.

11. Mir E, Palomo M, Rovira M, et al. Endothelial damage is aggravated in acute GvHD and could predict its development. Bone Marrow
Transplant. 2017;52(9):1317-1325

12. Haddad JG, Harper KD, Guoth $M$, et al. Angiopathic consequences of saturating the plasma scavenger system for actin. Proc Natl Acad Sci U S A. 1990;87(4):1381-1385.

13. Lee WM, Galbraith RM. The extracellular actin-scavenger system and actin toxicity. $\mathrm{N}$ Engl J Med. 1992;326(20):1335-1341.

14. Lind SE, Smith DB, Janmey PA, et al. Role of plasma gelsolin and the vitamin D-binding protein in clearing actin from the circulation. J Clin Invest. 1986;78(3):736-742

15. Schiodt FV, Ott P, Bondesen S, et al. Reduced serum Gc-globulin concentrations in patients with fulminant hepatic failure: association with multiple organ failure. Crit Care Med. 1997;25(8):1366-1370

16. Dahl B, Schiodt FV, Kiaer T, et al. Serum Gcglobulin in the early course of multiple trauma. Crit Care Med. 1998;26(2):285-289.

17. Chun RF. New perspectives on the vitamin D binding protein. Cell Biochem Funct. 2012;30(6):445-456.

18. Yamamoto N, Kumashiro R. Conversion of vitamin D3 binding protein (group-specific component) to a macrophage activating factor by the stepwise action of beta-galactosidase of B cells and sialidase of T cells. J Immunol. 1993; 151(5):2794-2802

19. Yamamoto N, Naraparaju VR. Vitamin D3binding protein as a precursor for macrophage activating factor in the inflammation-primed macrophage activation cascade in rats. Cell Immunol. 1996;170(2):161-167.

20. Kisker O, Onizuka S, Becker CM, et al. Vitamin $D$ binding protein-macrophage activating factor (DBP-maf) inhibits angiogenesis and tumor growth in mice. Neoplasia. 2003;5(1):32-40.

21. Jodele S, Davies SM, Lane A, et al. Diagnostic and risk criteria for HSCT-associated thrombotic microangiopathy: a study in children and young adults. Blood. 2014; 124(4):645-653.

22. Wallace G, Jodele S, Howell J, et al. Vitamin D deficiency and survival in children after hematopoietic stem cell transplant. Biol Blood Marrow Transplant. 2015;21(9):1627-1631.

23. Jodele S, Zhang K, Zou F, et al. The genetic fingerprint of susceptibility for transplant-associated thrombotic microangiopathy. Blood. 2016;127(8):989-996.

24. Ge L, Trujillo G, Miller EJ, et al. Circulating complexes of the vitamin $\mathrm{D}$ binding protein with G-actin induce lung inflammation by targeting endothelial cells. Immunobiology. 2014 219(3):198-207.

25. Kilpatrick LE, Phinney KW. Quantification of total vitamin-D-binding protein and the glycosylated isoforms by Liquid chromatographyisotope dilution mass spectrometry. J Proteome Res. 2017; 16(11):4185-4195.

26. Viau M, Constans J, Debray $\mathrm{H}$, et al. Isolation and characterization of the O-glycan chain of the human vitamin-D binding protein Biochem Biophys Res Commun. 1983; 117(1):324-331.

27. Borges CR, Rehder DS. Glycan structure of Gc protein-derived macrophage activating factor as revealed by mass spectrometry. Arch Biochem Biophys. 2016;606:167-179.

28. Lee WM, Galbraith RM, Watt $\mathrm{GH}$, et al. Predicting survival in fulminant hepatic failure using serum Gc protein concentrations. Hepatology. 1995;21(1):101-105.

29. Goldschmidt-Clermont PJ, Lee WM, Galbraith RM. Proportion of circulating Gc (vitamin D binding protein) in complexed form: relation to clinical outcome in fulminant hepatic necrosis. Gastroenterology. 1988;94(6):1454-1458.

30. Dahl B, Schiodt FV, Nielsen $M$, et al. Admission level of Gc-globulin predicts outcome after multiple trauma. Injury. 1999; 30(4):275-281.

31. Sun W, Kechris K, Jacobson S, et al. Common genetic polymorphisms influence blood biomarker measurements in COPD. PLoS Genet. 2016;12(8):e1006011.

32. Kirac D, Dincer Yazan C, Gezmis H, et al VDBP, VDR mutations and other factors related with vitamin $\mathrm{D}$ metabolism may be associated with Type 1 diabetes mellitus. Cell Mol Biol. 2018; 64(3):11-16.

33. Sedky NK, Hassanein SI, Gad MZ Independent assortment of GC gene polymorphism (rs2282679) and 25-hydroxyvitamin D levels in coronary artery disease. Can J Physio Pharmacol. 2018; 96(4):345-351.

34. Daffara V, Verdoia M, Rolla $R$, et al. Atherosclerosis Study G. Impact of polymorphism rs7041 and rs 4588 of Vitamin D binding protein on the extent of coronary artery disease. Nutr Metab Cardiovasc Dis. 2017 27(9):775-783 